\title{
Nonlinear Model Predictive Extended Eco-Cruise Control for Battery Electric Vehicles*
}

\author{
S. Amin Sajadi-Alamdari ${ }^{1}$, Holger $\operatorname{Voos}^{1}$, and Mohamed Darouach ${ }^{2}$
}

\begin{abstract}
Battery Electric Vehicles are becoming a promising technology for road transportation. However, the main disadvantage is the limited cruising range they can travel on a single battery charge. This paper presents a novel extended ecological cruise control system to increase the autonomy of an electric vehicle by using energy-efficient driving techniques. Driven velocity, acceleration profile, geometric and traffic characteristics of roads largely affect the energy consumption. An energy-efficient velocity profile should be derived based on anticipated optimal actions for future events by considering the electric vehicle dynamics, its energy consumption relations, traffic and road geometric information. A nonlinear model predictive control method with a fast numerical algorithm is adapted to determine proper velocity profile. In addition, a novel model to describe the energy consumption of a seriesproduction electric vehicle is introduced. The hyperfunctions concept is used to model traffic and road geometry data in a new way. The proposed system is simulated on a test track scenario and obtained results reveal that the extended ecological cruise control can significantly reduce the energy consumption of an electric vehicle.
\end{abstract}

\section{INTRODUCTION}

An Electric Vehicle (EV) has considerable advantages compare to the Internal Combustion Engine Vehicle (ICEV), such as higher efficiency, minor emissions, etc. Energy for the electric machine in Battery Electric Vehicle (BEV) is provided by an on-board chemical battery pack. Nevertheless, the BEV has a limited battery capacity which restricts cruising range. One of the universal methods which do not require any hardware technology improvement is to identify an energy-efficient profile. This method generally concerns to realise an optimised efficient profile either by training and giving hints to the driver or automating the velocity control.

The Ecological Cruise Control (Eco-CC) system with main objectives to reduce fuel consumption and air pollution have been extensively studied for ICEVs. An EcoCC system considering up-down road slopes with an ICEV fuel consumption model presented in [1]. This system derive an optimal vehicle control input by utilising a fast nonlinear model predictive control method. However, the traction boundaries of the vehicle which is a state dependent physical limit of the propulsion was not considered, although

\footnotetext{
*This work was supported by FNR "Fonds national de la Recherche" (Luxembourg) through AFR "Aides à la Formation-Recherche" Ph.D. grant scheme No. 7041503.

${ }^{1}$ Seyed Amin Sajadi Alamdari, and Holger Voos are with the Interdisciplinary Centre for Security, Reliability and Trust (SnT), University of Luxembourg, 6 rue Coudenhove-Kalergi, L-1359 Luxembourg, Luxembourg. e-mail: amin.sajadi@uni.lu

${ }^{2}$ Mohamed Darouach is with the Centre de Recherche en Automatique de Nancy (CRAN) UMR-CNRS 7039, Université de Lorraine, IUT de Longwy, 186 rue de Lorraine, F-54400 Cosnes et Romain, France.
}

a symmetric input constraint was considered. A predictive optimal velocity-planning algorithm that uses probabilistic traffic-signal phase and timing information to increase a vehicle's energy efficiency introduced in [2]. Nonetheless, dynamics of the vehicle was oversimplified which might not be able to represent realistic events. A broad review of recent research on factors affecting fuel consumption, Eco-Driving policy and the current state of art reviewed in [3], [4], [5].

On the other hand, little research to date has focused on the Eco-ADAS systems for BEVs. An optimal motion control problem with respect to BEV dynamics and its energy consumption formulated in [6]. However, road geometric information was not considered in this work. Closely related works are presented in [7], [8] which intend to minimize the energy consumption of a BEV considering with road and traffic information. However these are formulated in a simplified piecewise linear quadratic optimal control problem. In most of the presented literatures, the introduced systems were not capable of adapting the velocity to the road and traffic variations and the driver should take the velocity control back during different situations.

In this paper, a sophisticated Extended Eco-CC (Ext-EcoCC) system for BEVs is proposed in order to enhance the Advanced Driver Assistance System (ADAS). This system is capable of optimally control the velocity in road slopes, curves, and traffic speed limit variations without intervention of the driver. The main contributions of this paper are the following. First, a novel energy consumption model for a BEV is formulated and verified by measurements. Next, road geometry data including slope and curvature in addition with traffic speed limits are modelled by employing mathematical hyperfunctions. Finally, a finite nonlinear horizon optimal control problem is formulated and solved by adapting a real-time algorithm. Performance of the proposed system in terms of velocity profile, control input, comfort and safety index, and energy consumption on a test track is evaluated and analysed to appraise its capabilities for the ADAS framework.

The rest of this paper is organized as follows: The concept of an Ext-Eco-CC system, the BEV dynamics including its energy consumption model, road geometry as well as traffic model, and the nonlinear receding horizon control are introduced in Section II. Evaluation of the proposed system and its numerical simulation results are presented in Section III. Interpreted results and related discussion are given in Section IV. Conclusions and future work are given in Section V. 


\section{DEFINITIONS AND PROBLEM FORMULATION}

\section{A. Extended Ecological Cruise Control}

The concept of the proposed Ext-Eco-CC system for a $\mathrm{BEV}$ on a hilly road with road curves, and traffic speed limits is shown in Fig. 1. In this system, the driver set the fix reference velocity to drive and the traction input of a $\mathrm{BEV}$ is subjected to optimal control according to the longitudinal motion dynamics, energy consumption model, road geometry and traffic information. The Ext-Eco-CC system should generate a suitable velocity profile autonomously for the entire trip without requiring driver interventions. The approximate energy consumption of the vehicle can be calculated by a mathematical model. In addition, information of upcoming road slope, road curvature, and speed limit places can be modelled by position based functions. The control input is achieved based on the predicted driving state and relevant performance index which lead to a traction force input provides proper throttle and brake pedals manipulation.

\section{B. Electric Vehicle and Energy Consumption Dynamics}

The nonlinear longitudinal motion of a vehicle at time $t$ can generally be governed by the state equation

$$
\dot{\mathbf{x}}(t)=\mathbf{f}(\mathbf{x}(t), u(t)),
$$

where $\mathbf{x}(t)=[s(t), v(t), e(t)]^{T} \in \mathbb{R}^{3}$ denotes the state vector of travelled distance, velocity, and energy consumption or recovery of the vehicle correspondingly, and $u(t) \in \mathbb{R}$ is the control input. The vehicle is assumed to be a point mass at the center of gravity, and hence its acceleration along the longitudinal direction expressed by Newton's second law of motion

$$
d v(t) / d t=\left(F_{\text {trac }}(t)-F_{\text {res }}(t)\right) / M,
$$

where $M, F_{\text {trac }}(t)$, and $F_{\text {res }}(t)$ are the equivalent mass of the vehicle, the traction force, and the total motion resistive forces, respectively. Due to the property of the electric machine being able to cover a wide range of operating velocity, a single-gear transmission generally fulfils the vehicle performance requirement [9]. In a passenger vehicle, due to the rotational parts of the vehicle, an equivalent mass can be calculated by an empirical relation as $M=m\left(1+\delta_{1}+\delta_{2} i_{g}^{2}\right)$, where $m$ is the kerb mass of the vehicle, $\delta_{1}$ represents the total angular inertial moment of the wheels, $\delta_{2}$ serves as the

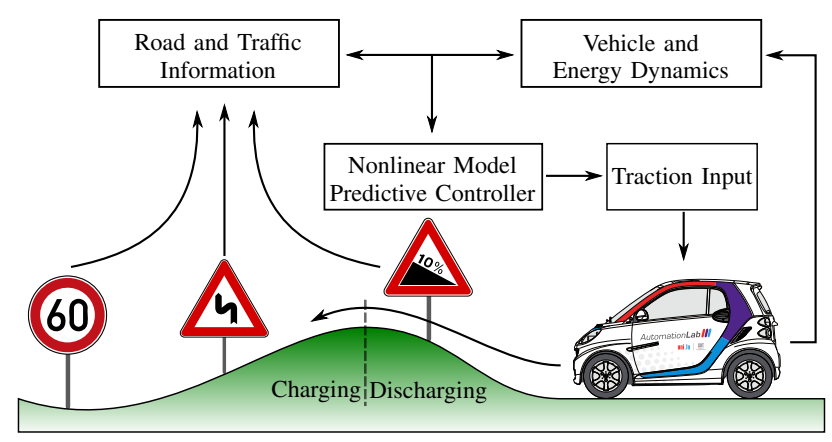

Fig. 1: Extended Ecological Cruise Control concept effect of the power-plant-associated rotating parts, and $i_{g}$ is the single transmission ratio [9].

The traction force depends on the equivalent mass and control input as $F_{\text {trac }}(t)=M u(t)$. A mechanical friction brake system accompanying regenerative brake is required to fulfil the brake performance which leads to a hybrid brake system. The total resistive aerodynamic drag, gradient, and rolling resistance forces can be represented by

$$
\begin{aligned}
F_{r e s} & =\frac{1}{2} \rho A_{f} C_{D} v^{2}+M g \sin \left(f_{s l p}(\theta(s))\right) \\
& +C_{r r}(v) M g \cos \left(f_{s l p}(\theta(s))\right),
\end{aligned}
$$

where $\rho, A_{f}, C_{D}, g, \theta(s)$, and $C_{r r}(v)$, are the air density, the vehicle frontal area, the aerodynamic drag coefficient, the gravitational acceleration, the road slope angle as a function of position (for more details, see [1]), and the velocity dependent rolling resistance coefficient, subsequently. The rolling resistance coefficient for passenger vehicles on a concrete road can be approximated as $C_{r r}(v)=0.01(1+v / 576)$ (for more details, see [9]).

Different approaches to model energy consumption of BEVs have been proposed (see e.g, [6]). Furthermore, the velocity and traction force have significant influence on the energy consumption. The energy consumption during cruising at constant speed is equitable to the resistive powers. This can be approximated through the curve-fit process with measurement data by a polynomial of velocity as $f_{\text {cruise }}=b_{3} v^{3}+b_{2} v^{2}+b_{1} v+b_{0}$. It is noteworthy that the $f_{\text {cruise }}$ formulation is adapted from [1]. The acceleration and deceleration, $a$, considering only the regenerative energy zone in the hybrid brake system can be approximated by a similar curve-fit process with measurement data using a polynomial of the control input as $f_{a}=a_{2} u^{2}+a_{1} u+a_{0}$. Therefore, combining $f_{\text {cruise }}$ and $f_{a}$, may lead to a model of the power consumption $p(t)$ in the BEV. At any given velocity and control input, a linear relation of the traction power-to-mass ratio $\left(p_{\text {trac }} / M\right)$ can be modelled as

$$
\dot{e}=p(t)=f_{a}\left(p_{\text {trac }} / M\right)+f_{\text {cruise }} .
$$

This novel model is capable of representing the regenerative braking effect when $u(t)<0$ for the full-range velocity and control input limits. This way, the power consumption of any BEV can be estimated by modelling traction-velocity characteristics map of the electric machine.

To conclude, the state equation (1) can be rewritten as

$$
\left[\begin{array}{c}
\dot{s} \\
\dot{v} \\
\dot{e}
\end{array}\right]=\left[\begin{array}{c}
v \\
u-\frac{1}{2 M} \rho A_{f} C_{D} v^{2}-g \sin \left(f_{s l p}(\theta(s))\right)-C_{r r}(v) g \cos \left(f_{s l p}(\theta(s))\right) \\
\left(a_{2} u^{2}+a_{1} u+a_{0}\right)(u v)+\left(b_{3} v^{3}+b_{2} v^{2}+b_{1} v+b_{0}\right)
\end{array}\right] .
$$

Note that, the $f_{s l p}(\theta(s))$ is a function of road slope profile from the start position to the end of the trip.

\section{Road Geometry and Traffic Models}

Knowledge of the road and traffic conditions ahead are beneficial for the ADAS safety and energy management applications. One way of representing the road geometric 
data is to define data points along the road centerline with specified intervals corresponding to the desired road profile accuracy level. There are several interpolation methods to construct new data points (see, e.g, [10], [11], [12]). However, the interval determination of data points can lead to either overestimated or underestimated accuracy levels for different road segments. Those approaches impose a tradeoff between accuracy level and response time of the models which may not be desirable for a real-time Eco-ADAS applications.

In this work, the road slopes, road curvatures, and traffic speed limits data are modelled as continuous and differentiable functions. These functions represent the data points in each segment of the road. The hyperfunction concept as a kind of generalized functions is used to interconnect the estimated segments of the road to each other at the boundaries. The road slope profile is proposed to be the sum of quadratic functions of position representing each road segments slope data. The modelling concept is shown in Fig. 2 .

Based on the stated context, the road slope profile can be defined as

$$
f_{s l p}(\theta(s)):=\sum_{i=1}^{N_{s g m}} H_{i}\left(s-s_{i-1}\right)\left(a_{i} s^{2}+b_{i} s+c_{i}\right) H_{i}\left(s-s_{i}\right),
$$

where $N_{s g m}$ is the number of road segments, the $H_{i}\left(s-s_{i-1}\right)$ and $H_{i}\left(s-s_{i}\right)$ are the hyperfunctions of the ith road segment. These hyperfunctions may be represented by the approximate Heaviside's functions at the boundary position values, $s_{i-1}$ and $s_{i}$, as follows

$$
\begin{aligned}
H_{i}\left(s-s_{i-1}\right) & =\frac{1}{2}\left(1+\tanh \left(k_{i+}\left(s-s_{i-1}\right)\right)\right), \\
H_{i}\left(s-s_{i}\right) & =\frac{1}{2}\left(1-\tanh \left(k_{i-}\left(s-s_{i}\right)\right)\right) .
\end{aligned}
$$

The road curvature and traffic speed limits profiles can be modelled in a similar way. The horizontal road curves may be parabolic or circular, which can be classified as simple, compound, reverse, and deviation curves. A simple curve has the same radius around a single arc of circle. Similar to the geometry of the Euler spiral, the simple curve is used to express the total absolute curvature profile in this work, which may be defined as

$$
f_{c r v}(\delta(s)):=\sum_{i=1}^{N_{c r v}} H_{i}\left(s-s_{\text {ent }}\right)\left|\frac{1}{R_{c r v_{i}}(s)}\right| H_{i}\left(s-s_{\text {ext }}\right),
$$

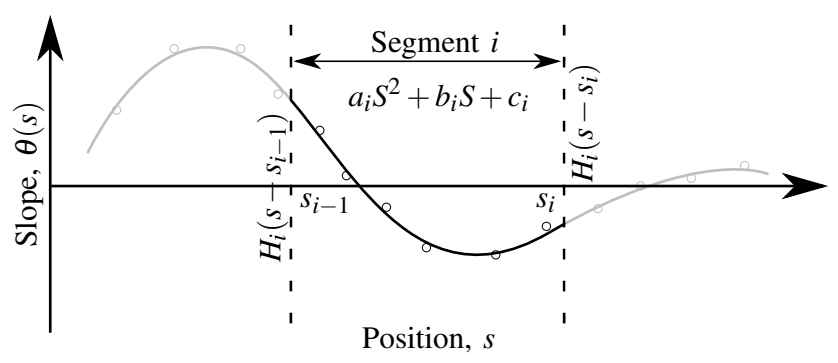

Fig. 2: Proposed road slope model at segment $i$ where $N_{c r v}$ is the number of road curves, and $R_{c r v_{i}}$ is the radius of a circle valid for the curve's arc length with two position points, $s_{\text {ent }}$ and $s_{\text {ext }}$, at the respective entrance and exit position of the curve $i$. The $R_{c r v}$ for a straight road segment can be considered as a large numerical number. The traffic speed limit places can be modelled as

$$
f_{l m t}(s):=\sum_{i=1}^{N_{l m t}} H_{i}\left(s-s_{s t r}\right)\left(v_{l m t}-v_{\text {max }}\right) H_{i}\left(s-s_{\text {end }}\right)+v_{\text {max }},
$$

where $N_{l m t}$ is the number of speed limit zones, and $v_{l m t}$ is the specified speed limit value at positions starts from $s_{\text {str }}$ upto the end of the zone $s_{\text {end }}$. The $v_{\max }$ is the maximum capable speed value of the vehicle. It should be noted that, the related $k_{i \pm}$ for the road slope, road curvature, and traffic speed limit models determine the smoothness of the transitions between segments and can be identified through curve-fit process. To conclude, the proposed method to model the road slope, road curvature, and speed limit profiles can be represented as continuous and differentiable functions which can be coupled with the vehicle dynamics and desired performance index.

\section{Nonlinear Model Predictive Control}

The traction input has limited traction power to accelerate or decelerate the vehicle at different velocity values. Exact equations also describing the boundaries of acceleration and hybrid braking systems are too complex containing unknown parameters (for more details, see [9]). Instead, the limits of the control input can be estimated by the vehicle's velocity-traction characteristics map. Hence, the control input is directly subject to these min-max inequality constraints $\left(u_{\min }(v) \leq u(t) \leq u_{\max }(v)\right)$. Due to the complexity of optimality conditions known as Karush-KuhnTucker (KKT) conditions, it is necessary to convert the inequality constraints into equality constraints by a penalty function method. The control input equality constraint can be expressed as

$$
C(\mathbf{x}, u)=\left(u-u_{a v r}(v)\right)^{2}-\left(u_{\max }(v)-u_{a v r}(v)\right)^{2}+u_{s l k}^{2}
$$

where $u_{s l k}$ is a slack variable, and $u_{\text {avr }}(v)=\frac{1}{2}\left(u_{\max }(v)+\right.$ $\left.u_{\min }(v)\right)$ is the average velocity depended of min-max control input boundaries.

In nonlinear model predictive control, for the given system dynamics (5), and the given input constraint (11), the following performance index with a finite horizon control problem should minimize the control input at each time, $t$. This can be expressed by

$$
\underset{u}{\operatorname{minimize}} J=\varphi\left(\mathbf{x}^{*}(T, t)\right)+\int_{0}^{T} L\left(\mathbf{x}^{*}(\tau, t), u^{*}(\tau, t)\right) d \tau
$$

where $\mathbf{x}^{*}(\tau, t)$ defines the state vector trajectory along the $\tau$-axis starting from $\mathbf{x}(t)$ as the initial state at $\tau=0$ over the prediction horizon $T$. The actuated control input is given by $u(t)=u^{*}(0, t)$ which is a state feedback control law. For more details about the nonlinear receding horizon control, see [13]. 
In this work, the terminal-cost function, $\varphi$ is chosen as

$$
\varphi=\frac{1}{2} q_{f}\left(e-e_{r e f}\right)^{2}
$$

where $e_{r e f}$ is reference energy consumption, and $q_{f}$ is the corresponding weight. The energy consumption, $e$, is only evaluated at the end of the prediction horizon. This helps to choose more flexible control actions independent of the integral-cost function, $L$, which can be defined as

$$
\begin{aligned}
L= & \frac{1}{2} q_{v}\left(v-v_{r e f}\right)^{2}+\frac{1}{2}\left(r_{u}\left(u-u_{r e f}\right)^{2}-q_{s l k} u_{s l k}\right) \\
& +\exp ^{\left(q_{c r v}\left(a_{\text {lat }}-a_{\text {lat.max }}\right)\right)} v^{2}+\exp ^{\left(q_{\text {lmt }}\left(v-f_{\text {lmt }}(s)\right)\right)} v
\end{aligned}
$$

where $u_{r e f}$ is the reference input, and $q_{v}, r_{u}$ are relative weightings. A small slack penalty, $q_{s l k}$, is added to avoid a singularity at $s_{s l k}=0$ and keep the control input away from the boundary of feasible set. In order to achieve a safe and comfortable ride during the road geometry variations, the lateral acceleration of the vehicle is defined as $a_{l a t}=$ $v^{2} f_{c r v}(\delta(s))$ and penalised in the cost function. An exponential function of the maximum allowable lateral acceleration, $a_{\text {lat.max }}$, with the related weight, $q_{c r v}$ is used for the lateral acceleration control. In addition, if the reference speed set by the driver is above the speed limit value, the velocity is penalised exponentially with the weight $q_{l m t}$.

For the optimal control problem composed of (5), (11), and (12), $H$ denotes the Hamiltonian defined as

$$
H(\mathbf{x}, \lambda, u, \mu):=L(\mathbf{x}, u)+\lambda^{T} \mathbf{f}(\mathbf{x}, u)+\mu^{T} C(\mathbf{x}, u),
$$

where $\lambda \in \mathbb{R}^{n}$ stands for the costate, and $\mu \in \mathbb{R}^{m_{c}}$ expresses the Lagrange multiplier related to the equality constraint. This can be solved efficiently by the Continuation and Generalized Minimal RESidual (C/GMRES) method introduced in [13]. This method is less computationally expensive and can be implemented in a real-time manner for the proposed ExtEco-CC system. The solution of the optimization problem is determined using the set of control inputs as initial guess solution with the measurements of the states. The first element of the set of control inputs is realised in the vehicle at each sampling time. Repeating this procedure keeps reproducing and updating the control input and lead to a close-loop nonlinear optimal control method, which is known as NMPC.

\section{SYSTEM EVALUATION}

\section{A. Numerical Simulation Configurations}

A Smart Electric Drive third generation which is available for experiments, is chosen here to model the dynamics of a BEV and its energy consumption. The parameters of the Smart ED dynamics model are derived from data sheets and real measurements as $m=975 \mathrm{~kg}, \delta_{1}=0.04, \delta_{2}=0.0025$, $i_{g}=9.922: 1, \rho=1.2041 \mathrm{~kg} / \mathrm{m}^{3}, A_{f}=2.05 \mathrm{~m}^{2}, C_{D}=0.37$, and $g=9.81 \mathrm{~m} / \mathrm{s}^{2}$. The main specifications of the Smart ED are summarised in [8] and [7].

In order to have a proper system identification of the Smart ED, dynamometer tests have been conducted (Fig. 3.) at Delphi Automotive Systems Luxembourg S.A. in

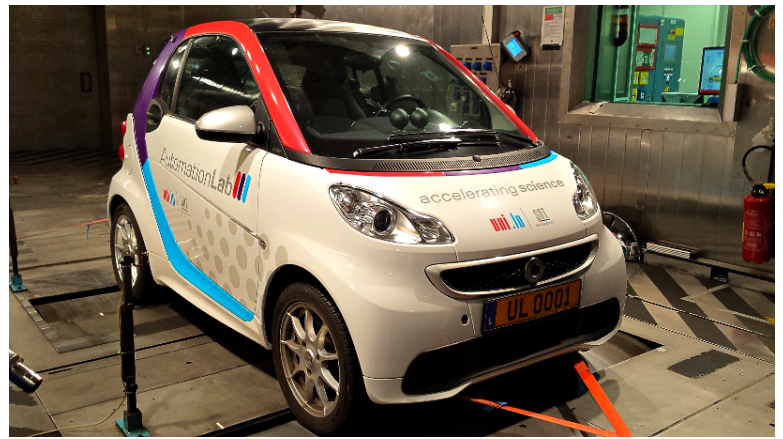

Fig. 3: Smart ED at the 4-wheel dynamometer test bench

Bascharage, Luxembourg [14]. The traction force, pedal positions, voltage and current of the battery at different velocity operating points are measured on a two-axles dynamometer and by reading the On-Board-Diagnosis (OBD) interface of the vehicle throughout the tests. These measurements help to model the overall dynamics and energy characteristics including electrical and mechanical characteristics of the vehicle. The proposed model for the energy consumption, (4), is identified as $a_{2}=0.01622, a_{1}=0.244, a_{0}=1.129$, $b_{3}=0, b_{3}=0, b_{2}=0.02925, b_{1}=0.257$, and $b_{0}=1.821$ with $98.46 \%$ coefficient of determination (R-squared). The limit $u_{\max }(v)$ can also be identified as the following relation

$$
u_{\max }(v)=c_{1}-c_{2} \tanh \left(c_{3}\left(v-c_{4}\right)\right) \text {, }
$$

where the constants are identified as $c_{1}=1.523, c_{2}=1.491$, $c_{3}=0.08751$, and $c_{4}=15.6$ with $99.74 \%$ coefficient of determination. The maximum hybrid brake system control input is chosen to be constant $\left(d_{1}=0\right), u_{\min }(v)=-5+d_{1} v$ $(\mathrm{N} / \mathrm{Kg})$, which is limited to a stable slip ratio region to avoid the wheels from locking up. Fig. 4. shows the experimentally identified power consumption map of the Smart ED with respect to the full-range control input and velocity. Each contour line represents the related power consumption (in $k W)$. At higher control input and velocity, the positive energy with higher rate is consumed. In contrast, at regenerative braking zone in different velocity, a limited amount of energy can be recovered.

A closed test track located at Centre de Formation pour

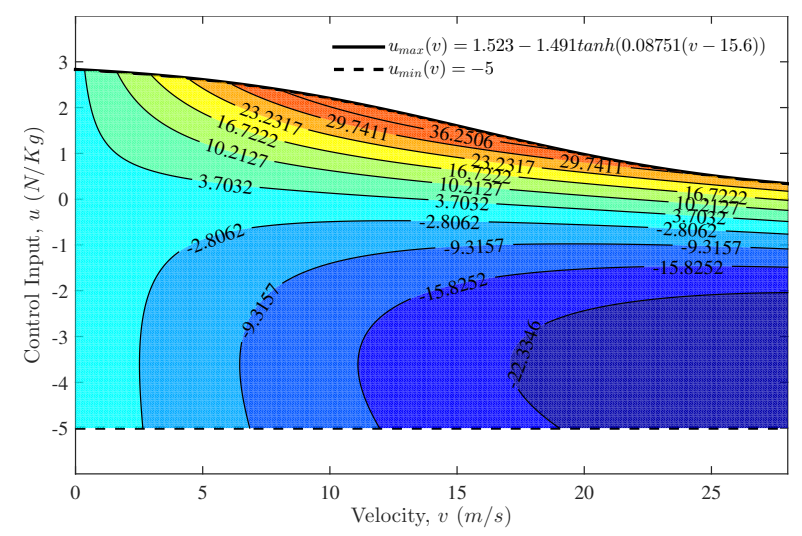

Fig. 4: Smart ED power consumption map 
Conducteurs S.A. Colmar-Berg, Luxembourg, is chosen to model the road geometry with assumed traffic information (Fig. 5.). The test track has a total length of $1.255 \mathrm{~km}$ and includes different turns and slopes. The altitude of the different points of the road is measured through the Global Positioning System (GPS) sensor with a resolution of $1 \mathrm{~m}$. The slope profile, $f_{s l p}(\theta(s))$, is fitted within nine segments with $98.93 \%$ coefficient of determination. This track has four main curves with $20 \mathrm{~m}, 25 \mathrm{~m}, 15 \mathrm{~m}$, and $27 \mathrm{~m}$ radius. The straight road segments are considered to have a nearly infinite radius. In addition, a speed limit $v_{l m t}=13.89 \mathrm{~m} / \mathrm{s}$ zone is assumed between positions $500 \leq s \leq 700$. Fig. 6 . shows the measured data with the proposed slope model, road curvature, and assumed speed limit profile formulated using (6), (9), and (10). A suitable prediction horizon $T=15 \mathrm{~s}$ is chosen to cover upcoming road and traffic events. This prediction horizon is discretized into $N=30$ steps of size $\Delta t=0.5 \mathrm{~s}$ based on the approximate vehicle's actuators maximum delay time. The total-cost function is set as $e_{r e f}=$ $0, q_{f}=0.25, v_{r e f}=25, q_{v}=1, u_{r e f}=F_{r e s}-M g \sin \left(f_{s l p}(s)\right)$, $r_{u}=20, q_{s l k}=20, q_{c r v}=1.2, a_{\text {lat.max }}=3.7$, and $q_{l m t}=0.1$. The maximum speed of the Smart ED is $v_{\max }=28 \mathrm{~m} / \mathrm{s}$ without activating the boost switch available in the vehicle. The weighting parameters are tuned manually by observing the performance in tracking the reference states considering the road and traffic information, safety and energy consumption in simulations.

\section{B. Simulation Results}

For the sake of a fair comparison, the proposed "Ext-EcoCC" system with the same initial conditions is compared to the proposed "Ext-CC" system without energy consumption

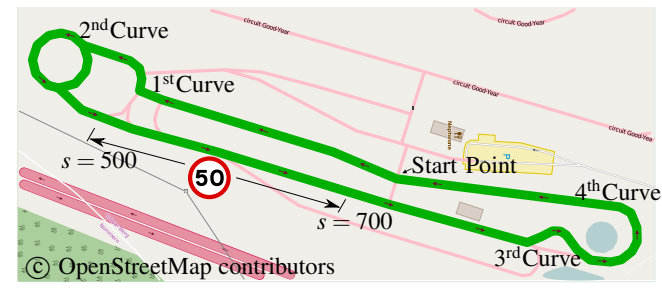

Fig. 5: Test track, Centre de Formation pour Conducteurs
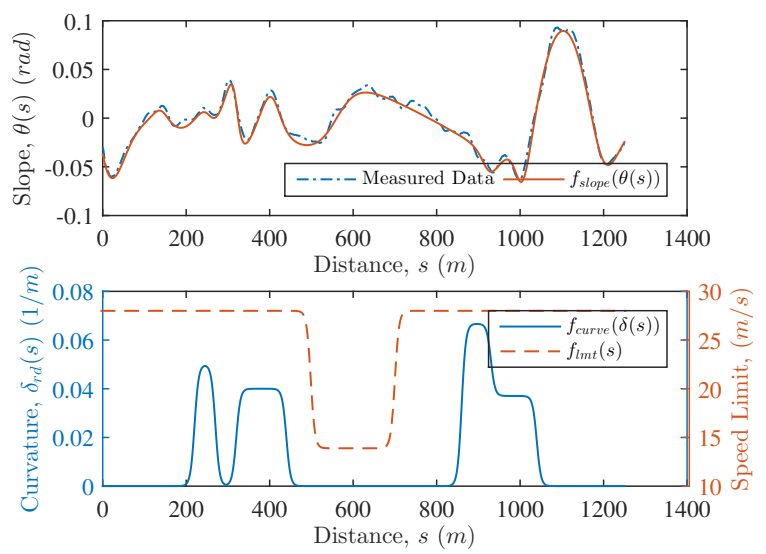

Fig. 6: Slope, Curvature, and Speed Limit profile at test track.

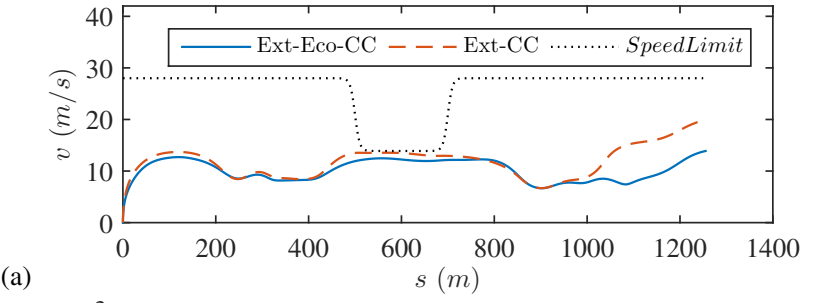

(a)

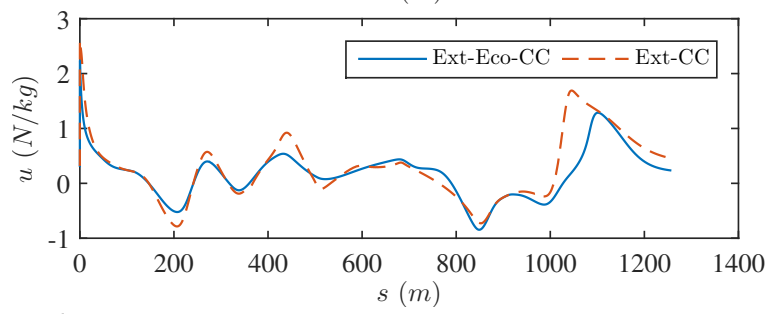

(b)
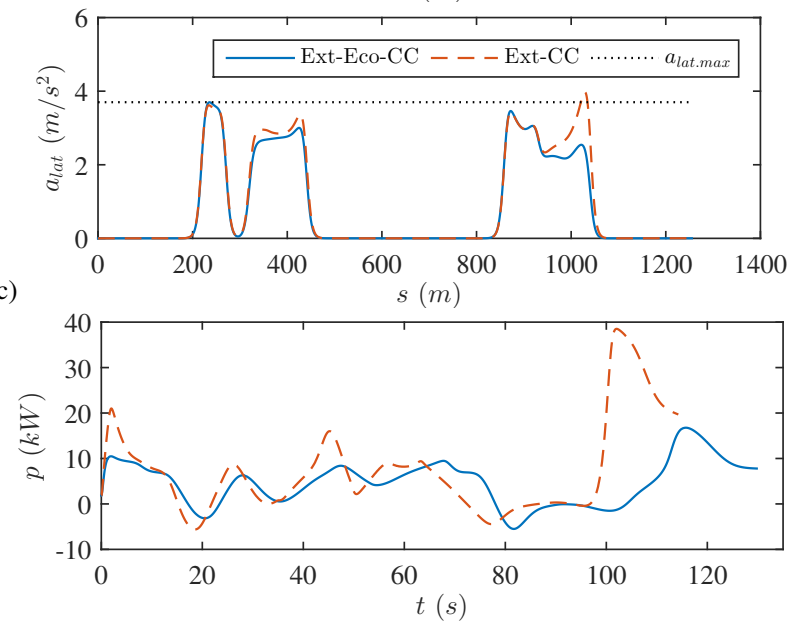

(d)

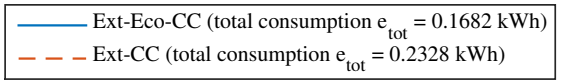

Fig. 7: Performance of the NMPC with Smart ED on the test track in terms of (a) velocity, (b) control input, (c) lateral acceleration, and (d) related power consumption with total energy consumption.

model $\left(q_{f}=0\right)$. The conventional Eco-CC and CC are not considering road curvature variations ans speed limit zones and therefore, these conventional systems may not be comparable with the proposed system.

Fig. 7. shows the optimal driving profile generated by the controller of the Ext-Eco-CC and Ext-CC system. Fig. 7a. shows the velocity profile at start point with initial standstill state. The controllers increase the velocity of the vehicle during straight down hill road segment and later reduce the velocity optimally as the vehicle approaches to the first and second curves. Next, the vehicle has to stay below the speed limit zone and afterwards does not speed up to reach the reference velocity due to the upcoming sharp third and forth curves. Comparing to the Ext-CC system, the Ext-Eco-CC system drives the vehicle much slower in the last segment of the road due to upcoming hilly road and thus saves a considerable amount of energy. The next graph, Fig. 7b. shows the related control input derived from the optimal control problem. The Ext-Eco-CC controller tries to avoid 
unnecessary aggressive control inputs namely strong braking and accelerations. Fig. 7c. shows the lateral acceleration of the vehicle in each curve remains below the reference maximum lateral acceleration value derived from a comfort and safety index. Note that since the driver controls the steering, the actual lateral acceleration of the vehicle in real driving test might be different. Fig. 7d. shows the related power consumptions profile on the test track and the final overall energy consumption, $e_{t o t}$, for the whole track. The Ext-Eco-CC takes any advantages of the road profile and traffic information to save as much energy as possible.

\section{DISCUSSION}

Previous studies show that it was more common to limit the traction input in order to reduce the energy consumption [1], and considering either none or partial road geometric [6] and traffic information [2] with simplified model and control problem formulations [8], [7]. These systems generally may provide suitable behaviour in some situations, however, the driver has to take back the velocity control of the vehicle in any other unforeseen situations. The overall direction of the obtained results showed that the proposed Ext-Eco-CC could be helpful to extend the limited cruising range of the BEV. This was achieved by reducing the driver interventions in velocity control and extending the autonomy of the vehicle with respect to road geometric and traffic information. For the simulation, it is found that the control input can be updated approximately every $1 \mathrm{~ms}$. Hence, this way of formulation should be a real-time capable controller for the proposed system.

It should be emphasized that with an increase of only $13 \%$ of travel time, the Ext-Eco-CC can save $27 \%$ of energy compared to the Ext-CC system at the test track. A balanced trade-off between the energy consumption and travel time can be achieved based on the driver's preference. It is found that the driver's high reference velocity was not possible to be achieved with ecological driving style in the test track. However, these achievements reveal the reliability of such Ext-Eco-CC system based on upcoming road and traffic information. The approximate energy consumption, road slope, road curvature, and speed limit models presented here are valid for the Smart ED and the test track profile. The parameters of these models and proper weights of the performance index can be applied to other electric vehicles and roads. Although this is a small study, the results can be generalised to urban and non-urban environments. The above presented features show that the proposed system might be useful in the intelligent transportation systems framework in terms of more semi-autonomous, safe, and energy efficient driving style.

\section{CONCLUSIONS AND FUTURE WORK}

A novel development of an advanced driver assistance system considering ecological driving style for the battery electric vehicles has been presented in this paper. This system has predictively determines a suitable velocity profile to extend the cruising range and the autonomy of the electric vehicle based on the road geometry and traffic information. It is shown that the vehicle has been controlled comfortably and safely confronted with different road and traffic speed limit situations.

In this paper, the Ext-Eco-CC system is only evaluated by the numerical simulation, however further real world experiments with the Smart ED will be carried out in the future. In addition, it would be desirable to include the functionalities of Adaptive Cruise Control (ACC) in the proposed concept. One of the sate-of-the-art challenges of the optimal control problem for the ACC system are stochastic phenomena in traffic. Investigating these challenges may be worthwhile to improve the Ext-Eco-CC system for the advanced driver assistance systems in the future.

\section{ACKNOWLEDGMENT}

The authors would like to acknowledge the Delphi Automotive Systems S.A., Centre de Formation pour Conducteurs S.A., T. Schwickart, and M. a. S. Kamal for their supportive and valuable comments in this study.

\section{REFERENCES}

[1] M. a. S. Kamal, M. Mukai, J. Murata, and T. Kawabe, "Ecological Vehicle Control on Roads With Up-Down Slopes," IEEE Transactions on Intelligent Transportation Systems, vol. 12, no. 3, pp. 783-794, sep 2011.

[2] G. Mahler and A. Vahidi, "An Optimal Velocity-Planning Scheme for Vehicle Energy Efficiency Through Probabilistic Prediction of TrafficSignal Timing," IEEE Transactions on Intelligent Transportation Systems, vol. 15, no. 6, pp. 2516-2523, dec 2014.

[3] E. Demir, T. Bekta, and G. Laporte, "A review of recent research on green road freight transportation," European Journal of Operational Research, vol. 237, no. 3, pp. 775-793, sep 2014.

[4] M. S. Alam and A. McNabola, "A critical review and assessment of Eco-Driving policy \& technology: Benefits \& limitations," Transport Policy, vol. 35, pp. 42-49, sep 2014.

[5] A. Sciarretta, G. De Nunzio, and L. L. Ojeda, "Optimal Ecodriving Control: Energy-Efficient Driving of Road Vehicles as an Optimal Control Problem," IEEE Control Systems, vol. 35, no. 5, pp. 71-90, oct 2015 .

[6] T. Wang, C. G. Cassandras, and S. Pourazarm, "Optimal motion control for energy-aware electric vehicles," Control Engineering Practice, vol. 38, pp. 37-45, may 2015.

[7] T. Schwickart, H. Voos, J.-R. Hadji-Minaglou, M. Darouach, and A. Rosich, "Design and simulation of a real-time implementable energy-efficient model-predictive cruise controller for electric vehicles," Journal of the Franklin Institute, vol. 352, no. 2, pp. 603-625, feb 2015 .

[8] T. Schwickart, H. Voos, J.-R. Hadji-Minaglou, and M. Darouach, "A Fast Model-Predictive Speed Controller for Minimised Charge Consumption of Electric Vehicles," Asian Journal of Control, vol. 18, no. 5, pp. n/a-n/a, 2015.

[9] M. Ehsani, Y. Gao, and A. Emadi, Modern Electric, Hybrid Electric, and Fuel Cell Vehicles: Fundamentals, Theory, and Design, Second Edition. CRC Press, 2009.

[10] D. Jeremie, "Navigation-Based Speed Profile Generation for an Open Road Speed Assistant," C. Anastasios, Ed., sep 2009, pp. 320-327.

[11] X. Yan, R. Zhang, J. Ma, and Y. Ma, "Considering Variable Road Geometry in Adaptive Vehicle Speed Control," Mathematical Problems in Engineering, vol. 2013, pp. 1-12, 2013.

[12] B. Kim and K. Yi, "Probabilistic and Holistic Prediction of Vehicle States Using Sensor Fusion for Application to Integrated Vehicle Safety Systems," IEEE Transactions on Intelligent Transportation Systems, vol. 15, no. 5, pp. 2178-2190, oct 2014.

[13] T. Ohtsuka, "A continuation/GMRES method for fast computation of nonlinear receding horizon control," Automatica, vol. 40, no. 4, pp. 563-574, apr 2004.

[14] "Delphi Automotive Systems Luxembourg S.A." [Online]. Available: http://www.delphi.com/ 